\begin{tabular}{|c|c|c|}
\hline Beitr. Ent. & Keltern & ISSN 0005-805X \\
\hline $\mathbf{5 1}(2001) 2$ & S. $341-354$ & 16.12 .2001 \\
\hline
\end{tabular}

\title{
Anthobium- und Deliphrosoma-Arten und -Unterarten der Forschungs- und Sammelreise 2000 nach Bulgarien und Anmer- kungen zu zwei anderen Arten der Balkanhalbinsel
}

\author{
(Coleoptera: Staphylinidae: Omaliinae) ${ }^{1}$
}

Mit 7 Figuren

LOTHAR ZERCHE

\section{Zusammenfassung}

Antbobium belasizaense sp. n. aus dem Belasiza-Gebirge, Delipbrosoma slaniankaense sp. n. aus dem SlaviankaGebirge, beide in Südwest-Bulgarien, und Delipbrosoma prolongatum komense subsp. n. aus dem Kom-Massiv des Balkan-Gebirges in Nordwest-Bulgarien werden beschrieben. Lathrimaeum kuehnelti SCHEERPELTZ, 1963 syn. n. ist ein Synonym von Antbobium melanocephalum (LLLGER, 1794). Latbrimaeum knappei SCHEERPELTZ, 1961 syn. n. ist ein Synonym von Anthobium ganglbaueri (LUZE, 1905). Antbobium melanorephalum (ILLIGER, 1784) wird zum ersten Mal aus Bulgarien gemeldet. Deliphrosoma mandli (SCHEERPELTZ, 1937) wurde zum ersten Mal sowohl im Weschen-Massiv als auch im Kom-Massiv des Balkan-Gebirges nachgewiesen. Deliphrosoma bulgaricum ZERCHE, 1998, beschrieben aus dem Rila-Gebirge, wird auch aus dem Midschur-Massiv des Balkan-Gebirges gemeldet. Für Deliphrosoma prolongatum australe ZERCHE, 1998, Antbobium atrocepbalum (GYLLENHAL, 1827) und Antbobium ganglbaueri (LUZE, 1905) wird weiteres Material mitgeteilt. Ein Katalog der bulgarischen Delipbrosoma-Arten und -Unterarten wird vorgelegt. Es werden Anmerkungen zu Deliphrosoma angulatum ASSING \& WUNDERLE, 2001 and Delipbrosoma fratellum (ROTTENBERG, 1874) gemacht und für beide Arten zusätzliche Funde mitgeteilt. Deliphrosona angulatum wird zum ersten Mal aus dem Iti-, dem Peristeri- und dem Timfi-Massiv gemeldet.

\section{Summary}

Anthobium belasizaense sp. $\mathrm{n}$. from the Belasiza mountain tange, Deliphrosoma slaviankaense sp. n. from the Slavianka mountain range, both in southwestern Bulgaria, and Delipbrosoma prolongatum komense subsp. $\mathrm{n}$. from the Kom massif of the Balkan mountain range in northwestern Bulgaria are described. Latbrimaeum kuebnelti SCHEERPELTZ, $1963 \mathrm{syn}$. $\mathrm{n}$. is a synonym of Antbobium melanocephalum (ILLIGER, 1794). Latbrimaeum knappei SCHEERPELTZ, 1961 syn. n. is a synonym of Antbobium ganglbaueri (LUZE, 1905). Anthobium melanocephalum (ILLTGER, 1784) is reported from Bulgatia for the first time. Deliphrosoma mandli (SCHEERPELIZ, 1937), known from the Botev massif of the Balkan mountain range and from the Vitosha mountain range, is reported for the first time for both the Veshen massif and the Kom massif of the Balkan mountain range. Delipbrosona bulgaricum ZERCHE, 1998, described from the Rila mountain range, is reported for the first time from the Midshur massif of the Balkan mountain range. For Delipbrosoma prolongatum australe ZERCHE, 1998, Anthobium atrocephalum (GYLENHAL, 1827), and Antbobium ganglbaueri (LuZE, 1905) further material is reported. A catalogue of the Bulgarian Deliphrosoma species and subspecies is presented. Deliphrosoma angulatum ASSING \& WUNDERLE, 2001 and Delipbrosoma fratellum (ROTTENBERG, 1874) are commented and additional records are presented. Deliphrosoma angulatum is reported for the first time from the Iti, Peristeri, and Timfi massifs.

\footnotetext{
1 Das neue Anthobium- und Delipbrosoma-Material aus Bulgarien sowie Teile des Materials aus Gtiechenland wurden auf Reisen gesammelt, die durch eine Sachbeihilfe der Deutschen Forschungsgemeinschaft gefördert wurden (ZE 301/5-1).
} 


\section{Einleitung}

Eine Forschungs- und Sammelreise nach Bulgarien vom 2.-30. Mai 2000 (ZERCHE \& BEHNE), bei der mit Ausnahme der Rhodopen alle Hochgebirge und Bergmassive über $1900 \mathrm{~m}$ Höhe aufgesucht wurden und dabei stets am Rande der Schneefelder gesammelt wurde, führte zu ganz überraschenden Funden einer neuen Art der Gattung Anthobium LEACH, 1819 und von zwei neuen Arten bzw. Unterarten der Gattung Deliphrosoma REITTER, 1909.

Die bulgarischen Gebirge sind noch immer unzulänglich erforscht - insbesondere die früher unzugänglichen Grenzgebirge -, obwohl es sich um die achte Reise des Autors in die bulgarischen Hochgebirge gehandelt hat, deren bisherige Ergebnisse über die Gattung Deliphrosoma in fünf Publikationen eingeflossen sind (ZERCHE 1991a, b, 1997, 1998, 1999).

\section{Danksagung}

LUTZ BEHNE, Eberswalde, und OGNJAN ILIEV, Biologische Fakultät der Universität Sofia (BFUS), waren beim Sammeln in den bulgarischen Hochgebirgen beteiligt. OGNJAN ILIEV sorgte auch für die noch immer erforderliche Erlaubnis zum Besuch der Grenzgebirge zu Griechenland. Ein namentlich nicht bekannter Grenzsoldat, der uns im Belasiza-Gebirge begleitet hat, wat ein ortskundiger Führer. WERNER MARGGI, Thun, schulde ich Dank für das Überlassen von Material von zwei Arten aus Griechenland. HARALD SCHILlHAMMer, Naturhistorisches Museum Wien (NHMW), danke ich für die Ausleihe von Typen. Frau BIANKA KatNig, Eberswalde, fertigte die Abbildungen in Tusche. CHRISTIAN KuTZSCHER, Eberswalde, danke ich für die Anfertigung der Habitus-Abbildungen. VOLKER AsSING, Hannover, stellte mir vorab die Beschreibung von Deliphrosoma angulatum ASSING \& WUNDERLE, 2001 mit ihren Abbildungen zur Verfügung.

\section{Anthobium belasizaense sp. n.}

Fig. 1, 5

\section{Typenmaterial}

Holotypus (đ): BG: Belasiza S Petritsch, Malak Kongur, N-Hang, $1780 \mathrm{~m}$, Schneefeld in Wiese, unter Steinen, 41 $19^{\prime} 56$ N 2310'30 O, Gesiebe, 7.V.2000, leg. Zerche / HOLOTYPUS Anthobium belasizaense Zerche (DEI).

Paratypen: (1 ơ, 1 \&): BG: Belasiza S Petritsch, Malak Kongur, N-Hang, 1750 m, Schnee-

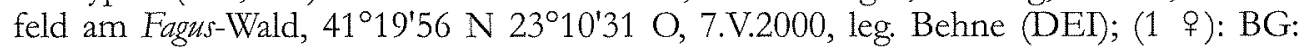
Belasiza S Petritsch, Malak Kongur, N-Hang, $1710 \mathrm{~m}$, Schneefeld in Juniperus, Gesiebe, 4120'04 N 2310'37 O, 7.V.2000, leg. Zerche; (1 o ): BG: Belasiza S Petritsch, Malak Kongur, N-Hang, $1780 \mathrm{~m}$, Schneefeld in Wiese, unter Steinen, 41 $19^{\prime} 56$ N $23^{\circ} 10^{\prime} 30 \mathrm{O}$, 7.V.2000, leg. O. Iliev (BFUS).

\section{Beschreibung}

Maße des Holotypus [in mm]: Kopfbreite 0,70; Augenlänge (im Profil) 0,17; Schläfenlänge (im Profil) 0,10; Pronotumlänge 0,62; Pronotumbreite 1,04; Nahtlänge 1,26; Elytrenbreite 1,32; Abdomenbreite 1,25.

Körper rotbraun. Kopf dunkler rotbraun bis pechbraun, Ocellen und Mundteile rotbraun. Antennen zur Spitze schwach verdunkelt. Punktur des Kopfes relativ fein, aber deutlich. Punktur des Pronotums deutlich stärker, aber etwas schwächer als auf den Elytren. Punktur der Elytren ziemlich stark, unregelmäßig gereiht. Kopf neben den Augen mit etwas stärkerer Mikroskulptur, der übrige Körper ohne Mikroskulptur, stark glänzend. Größe: 2,87-3,64 mm (Mittelwert: 3,3 [n = 4]). 


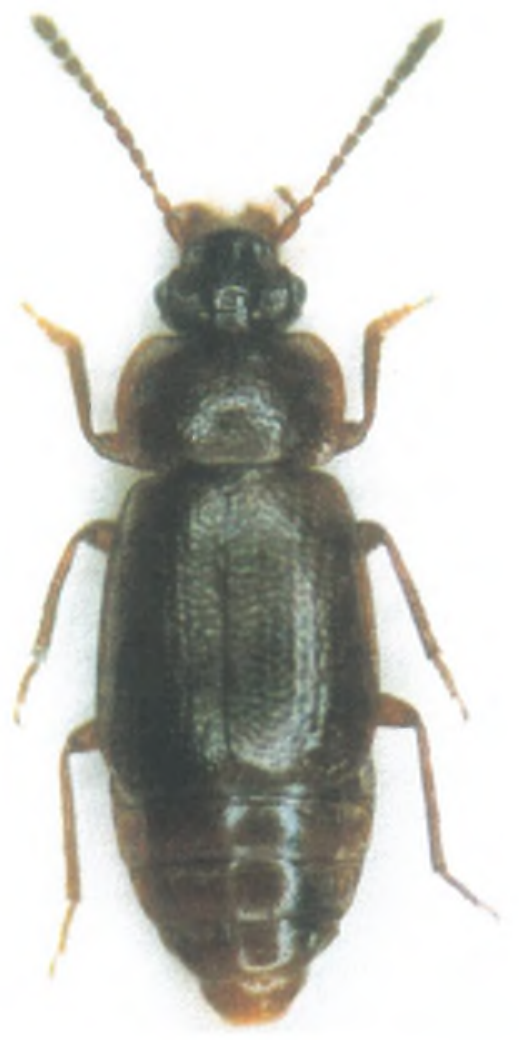

Fig. 1. Antbobium belasizaense sp. $\mathrm{n}$. [Holotypus].

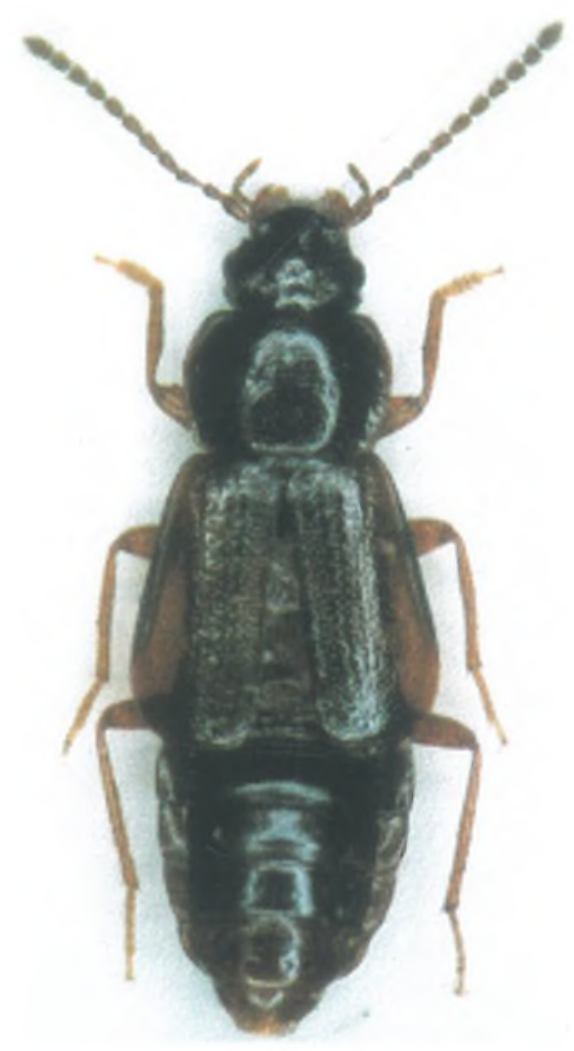

Fig. 2. Deliphrosoma slaviankaense sp. $\mathrm{n}$. [Holotypus].

Augen relativ groß und vorgewölbt, viel länger als die Schläfen (1,7). Ocellen mäßig groß, etwas hinter dem Niveau des Augenhinterrandes, voneinander weiter entfernt als jeweils vom Auge $(1,33)$. Kopf nicht durch eine Furche vom Hals getrennt. Scheitel mit flachen Schrägeindrücken vor den Ocellen. Kopfrand über den Fühlerbasen wulstförmig erhaben; dahinter mit flacher Einkerbung. Mitte des Scheitels gleichmäßig und ohne jede Begrenzung in den Clypeus übergehend.

Pronotum stark quer $(1,68)$, etwa in der Mitte am breitesten; nach vorn kaum stärker als nach hinten verengt. Seitenrand vor den abgerundeten Hinterwinkeln nicht ausgeschweift. Vorderwinkel etwas breiter verrundet als die Hinterwinkel. Vorderrand flach doppelbuchtig. Hinterrand gerade bis sehr flach konvex. Seitenrandeindrücke ziemlich flach. Mitte des Pronotums gleichmäßig gewölbt, Seiten breit gekehlt.

Elytren mäßig gestreckt, deutlich erweitert, über der breitesten Stelle am Ende des vierten Fünftels viel breiter $(1,35)$ als über den etwas reduzierten Schultern; Schultern schmaler als das Pronotum. Naht kaum kürzer als die größte Breite $(0,95)$. Hautflügel fehlen.

Abdomen ziemlich breit, über dem Segment IV am breitesten, nach hinten stark verengt. Tomentflecke fehlen. Hinterrand des Tergits VII mit sehr feinem Fransensaum. 
$0^{\pi}$ : Vordertarsen relativ stark erweitert; Segment II etwas schmaler als das Distalende der Vorderschienen; Segment III erkennbar schmaler, Segment IV viel schmaler als das Segment II. Vorderschienen vor der Mitte des Innentandes erweitert, zum Apex flachbogig ausgeschnitten. Mittelschienen distal nur schwach erweitert, körnchenartige Setae [,peglike setae" sensu HAMMOND (1972)] sehr schwach ausgebildet. Aedoeagus relativ groß und breit, ein Viertel $(0,24-0,25)$ der Körperlänge messend (Fig. 5). Parameren schmal und ziemlich kurz, den Apex des Medianlobus nicht erreichend, dem Medianlobus anliegend.

\section{Differentialdiagnose}

Bei dem in Bulgarien sehr häufigen Anthobium atrocephalum sind die Seitentänder der Elytren fast gleichmäßig gebogen, seine Elytren sind kurz nach der Mitte am breitesten. Das in diesem Merkmal ähnliche Antbobium melanocephalum ist zusätzlich deutlich größer, flacher und hat scharfe Pronotum-Hinterwinkel. Antbobium ganglbaneri ähnelt in der Körperform atrocephatum, ist aber meist deutlich heller gefärbt und durch seine schwach krenulierten Pronotum-Seitenränder gut charakterisiert. Die weit verbreiteten Arten $A$ nthobium unicolor (MARSHAM, 1802) und Antbobium fusculum (ERICHSON, 1839) können aus chorologischen Gründen ausgeschlossen werden, beide sind von der Balkanhalbinsel unbekannt (HORION 1963).

Anthobium belasizaense sp. $\mathrm{n}$. ist von allen Antbobium-Arten durch seine schwach entwickelten Schultern, die Schulterbreite ist deutlich geringer als die Pronotumbreite, die nach hinten stärker erweiterten Elytren mit ihrer breitesten Stelle am Beginn des hinteren Fünftels, die flache Wölbung der Elytren sowie das Fehlen der Hautflügel zu unterscheiden. Weil der locus typicus der neuen Art im Belasiza-Gebirge am Dreiländereck zwischen Bulgarien, Griechenland und Mazedonien liegt, waren insbesondere zwei bisher nicht revidierte Arten zu prüfen, die SCHEERPELTZ $(1961,1963)$ aus Mazedonien bzw. Griechenland beschrieben hat. Beide Namen erwiesen sich als Synonyme [siehe Anthobium ganglbaueri (LUZE, 1905) bzw. Anthobium melanocephalum (ILLIGER, 1794)].

\section{Verbreitung}

Die neue Art ist nur vom locus typicus in der oberen montanen/subalpinen Region des Belasiza-Massivs in Südwest-Bulgarien bekannt. Die verschiedenen Fundpunkte liegen nur wenige Hundert Meter auseinander. Sie sind nur etwa $1 \mathrm{~km}$ von der griechischen Grenze (griechischer Name: Kerkíni Óros) und nur etwa $15 \mathrm{~km}$ von der mazedonischen Grenze entfernt.

Derivatio nominis: Den Namen belasizaense (Adjektiv) wähle ich, weil die neue Art wahrscheinlich im Belasiza-Gebirge endemisch ist.

Untersuchte Exemplare: $20^{\pi} \sigma^{\star}, 3$ 우요.

\section{Anthobium melanocephalum (ILLIGER, 1794)}

Silpha melanocepbala ILLIGER, 1794: 596.

- Lathrimaeum kuebnelit SCHEERPELTZ, 1963: 426. syn. n.

\section{Revision des Holotypus von Lathrimaeum kuehnelti}

Holotypus (o): o / Ano Trikala / Tannenwald 21.IV.60 / Peloponnes / Griechenland IV.1960 W. Kühnelt / Lathrimaeum Kühnelti m. / TYPUS Lathrimaeum Kühnelti O. Scheerpeltz / ex coll. Scheerpeltz / Anthobium melanocephalum (lliger, 1794) det. Zerche 2000 (NHMW). 
Lathrimaeum kuebnelti SCHEERPELTZ, 1963 syn. n. erweist sich als Synonym der weit verbreiteten und in den griechischen Gebirgen, auch im Killini, sehr häufigen Art Antbobium melanocephalum (ILLIGER, 1794). Der weibliche Holotypus weist keinerlei Besonderheiten auf; das Exemplar ist charakteristisch für Anthobium melanocephalum.

\section{Material}

Slavianka (Ali-Botusch), S Goleschovo, NW Mt. Gozev Vrach, N-Hang, 1955 m, Schnee-

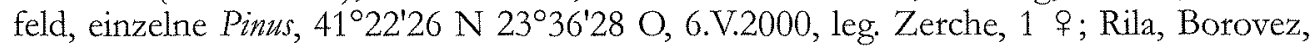
oberh. Sitnjakovo, 1965 m, N-Hang, letzte Schneereste im Picea-Wald, 42¹4'11 N $23^{\circ} 37^{\prime} 29$ O, 14.V.2000, leg. Zerche \& Behne, 5 우; Vitoscha, Aleko, 1835 m, Gesiebe am Schnee-

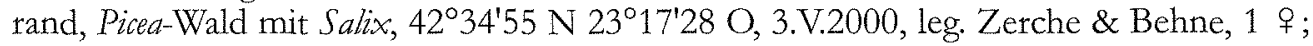
Malescherska Planina, oberh. Gorna Bresniza, $1650 \mathrm{~m}$, Senke, N-Hang, Fagus-Wald,

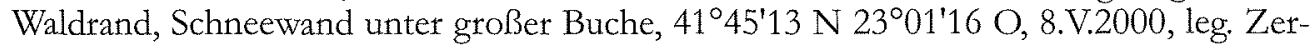
che, 10 ; Stara Planina, Midschur-Massiv, N-Seite, S Gorni Lom, Schneerinne, $1640 \mathrm{~m}$,

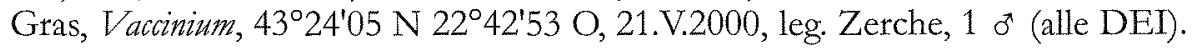

\section{Verbreitung}

Anthobium melanocephalum ist in Europa weit verbreitet. In den Gebirgen Griechenlands ist die Art sehr häufig. Ihr bulgarisches Areal beschränkt sich nach bisheriger Kenntnis auf die südwestlichen und westlichen Gebirge. Wir haben sie im Jahr 2000, bei der achten Reise, zum ersten Mal selbst gefunden. Sie war mir aber schon durch einen Fund aus dem Rila-Gebirge östlich Blagojevgrad, Urwald „Parangalitza“, 10.X.1989, 5 Ex., leg. O. ILIEV (BFUS; DEI), bekannt. Möglicherweise hat das ,seltenere" Vorkommen in Bulgarien nur phänologische Gründe. In Bulgarien wurde bisher stets im Juni/Juli gesammelt, in Griechenland dagegen im März/April. Erstmeldung für Bulgarien!

\section{Anthobium atrocephalum (GYLLENHAL, 1827)}

\section{Material}

Slavianka (Ali-Botusch), S Goleschovo, NW Mt. Gozev Vrach, O-Hang, $2030 \mathrm{~m}$, Schneefeldrand, Gesiebe, einzelne Pinus, Juniperus, 41 $23^{\prime} 07$ N $23^{\circ} 36^{\prime} 35$ O, 6.V.2000, leg. Zerche \& Behne, 5 o $\sigma^{*}, 3$ o ; ; S-Pirin, Orelek, O-Hang, $1560 \mathrm{~m}$, Pinus-Wald mit Junipe-

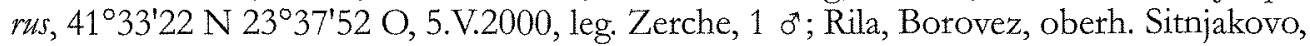
1965 m, N-Hang, letzte Schneereste im Picea-Wald, 42¹4'11 N 2337'29 O, 14.V.2000, leg. Zerche \& Behne, 1 o, 1 \%; Maleschevska Planina, oberh. Gorna Bresniza, 1650 m,

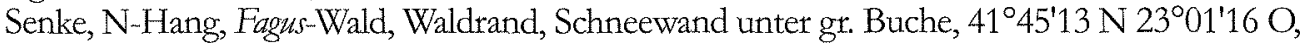
8.V.2000, leg. Zerche, $20^{*}, 1$ \& Stara Planina, Midschur-Massiv, N-Seite, S Gorni Lom,

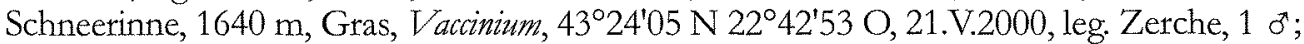
Stara Planina, Midschur-Massiv, N-Seite, S Gorni Lom, Schneerinne, $1675 \mathrm{~m}$, Juniperus,

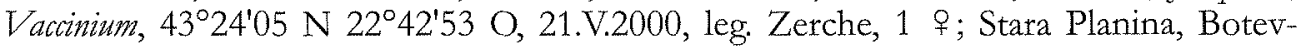
Massiv, N-Seite, Kar an der Pobita Glava, $1860-1900 \mathrm{~m}$, Schneefeld, Seitenkante, $42^{\circ} 43^{\prime} 51$ N 245'ㄹ O, 27.V.2000, leg: Zerche, $10^{*}$ (alle DEI).

\section{Verbreitung}

Die Art ist weit verbreitet und in Europa in Wäldern sehr häufig. Das gilt auch für Bulgarien; es wurden nur wenige Belegexemplare mitgenommen. 


\section{Anthobium ganglbaueri (LUZE, 1905)}

Latbrimaeum ganglbaueri LUZE, 1905: 65.

= Latbrimaeum knappei SCHEERPELTZ, 1961:89, syn. $\mathbf{n}$.

\section{Revision der Typenserie von Lathrimaeum knappei und Designation eines Lectotypus}

Lectotypus ( $\left.0^{\star}\right): 0^{*} /$ Dr. F. und M. Knappe - 15.VII.1960 / Gebirgszug der Galičica, 1000-1200 m Buchenwald / Yugoslavien Ramme südöstl. des Ochrida-Sees / TYPUS Lathrimaeum Knappei O. Scheerpeltz / ex coll. Scheerpeltz / LECTOTYPUS Lathrimaeum knappei Scheerpeltz, 1961 / Anthobium ganglbaueri (Luze, 1905) det. Zerche 2000 (NHMW). Hiermit designiert!

Paralectotypen $\left(10^{x}, 2\right.$ 우): 1 ㅇ, TYPUS, $10^{x}, 1$ ㅇ, COTYPUS, sonst wie Lectotypus (NHMW).

Ein Lectotypus wird zur Stabilisierung des Namens designiert, weil SCHEERPELTZ keinen Holotypus festgelegt hat. Seine Bezeichnung "Typus" ist gegenstandslos, weil er sie gleichberechtigt für zwei Tiere der Typenserie verwendet hat.

Latbrimaeum knappei SCHEERPELTZ, 1961 syn. n. erweist sich als Synonym der auf der Balkanhalbinsel weit verbreiteten und häufigen Art Anthobium ganglbaueri (LUZE, 1905). Die Typenserie weist keinerlei Besonderheiten auf; es handelt sich um charakteristische Anthobium ganglbaueri-Exemplare.

\section{Material}

Belasiza S Petritsch, Hütte Belasiza, $720 \mathrm{~m}$, Gesiebe, Castanea-Fagus-Wald, $41^{\circ} 22^{\prime} 11 \mathrm{~N}$ $23^{\circ} 11^{\prime} 13$ O, 5.V.2000, leg. Behne, $10^{\star}$ (DEI); Konjavska Planina N Kjustendil, Viden-

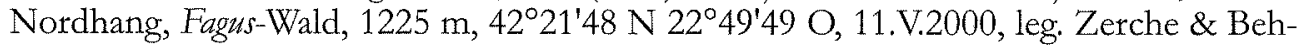
ne, 1 of (DEI).

\section{Verbreitung}

Die Art wurde aus Herkulesbad (Bâile Herculane) in den rumänischen Südwest-Karpaten beschrieben. Sie ist auch aus Griechenland und Mazedonien bekannt. In Bulgarien ist sie in niederen und mittleren Berglagen weit verbreitet. Sie wurde auch bei Vlas in der Eminska Planina am Schwarzen Meer, 12., 13. und 15.V.1987, leg. BEHNE \& HEINIG (DEI), nachgewiesen.

\section{Deliphrosoma slaviankaense $\mathrm{sp} . \mathrm{n}$.}

Fig. 2, 6

\section{Typenmaterial}

Holotypus ( $0^{\star}$ ): BG: Slavianka (Ali-Botusch), S Goleschovo, NW Mt. Gozev Vrach, OHang, $2030 \mathrm{~m}$, Schneefeldrand, Gesiebe, einzelne Pinus, Juniperus, 41 ${ }^{\circ} 23^{\prime} 07$ N $23^{\circ} 36^{\prime} 35$ O, 6.V.2000, leg. Zerche \& Behne $\backslash$ HOLOTYPUS Deliphrosoma slaviankaense Zerche (DEI).

Paratypus $\left({ }^{\star}\right)$ : wie Holotypus (DEI).

\section{Beschreibung}

Maße des Holotypus [in mm]: Kopfbreite 0,76; Augenlänge (im Profil) 0,17 ; Schläfenlänge (im Profil) 0,17; Pronotumlänge 0,76; Pronotumbreite 1,12; Nahtlänge 1,37; Elytrenbreite 1,40; Abdomenbreite 1,40. 
Kopf, Fläche des Pronotums, Scutellum und Abdomen mit Ausnahme der Spitze geschwärzt. Pronotum an den Seitentändern schmal rotbraun gerandet. Elytren und Abdomenspitze düster rotbraun. Mundteile, Ocellen und Beine etwas heller rotbraun. Antennenbasis \pm rotbraun: Scapus heller oder dunkler rotbraun, Basalteil des Pedicellus und des Segments III rotbraun; übrige Antennensegmente verdunkelt. Punktur auf Kopf und Pronotum relativ fein, auf den Elytren deutlich stärker und teilweise unregelmäßig gereiht. Abdomen fein und zerstreut punktiert; sehr fein rundmaschig chagriniert.

Größe: 4,48-4,90 mm.

Augen ziemlich klein und nur flach vorgewölbt, bei Ansicht im Profil so lang wie die Schläfen. Ocellen klein, kurz hinter dem Niveau des Augenhinterrandes, voneinander viel weiter entfernt als jeweils vom Auge $(1,48)$. Kopf nicht durch eine Furche vom Hals getrennt. Scheitel mit flachen Schrägeindrücken vor den Ocellen, ohne Mitteleindruck. Kopfrand über den Antennenbasen nur flach wulstförmig erhaben; dahinter mit kleiner, aber tiefer Einkerbung. Mitte des Scheitels gleichmäßig und ohne jede Begrenzung in den Clypeus übergehend.

Pronotum stark quer $(1,47)$, deutlich vor der Mitte am breitesten; nach vorn nur mäßig verengt, die Vorderwinkel nur kurz abgerundet; nach hinten etwas konvex gebogen, nicht ausgeschweift; Hinterwinkel angedeutet. Vorderrand sehr flach doppelbuchtig. Hinterrand sehr flach konvex. Seitenrandeindrücke flach. Mitte des Pronotums vor dem Hinterrand mit einem kaum erkennbaren Eindruck (ohne ankerartige Vertiefung).

Elytren ziemlich gestreckt und deutlich erweitert; ihre Schultern erkennbar schmaler als das Pronotum (0,92); ihre breiteste Stelle am Beginn des letzten Drittels. Naht etwa so lang wie die größte Breite (0,98-1,06). Hautflügel verkürzt.

Abdomen schmal, über dem Segment IV am breitesten, nach hinten stark verengt. Tergite ohne Tomentflecke. Hinterrand des Tergits VII ohne Fransensaum.

$\sigma^{*}$ : Vordertarsen stark erweitert; Segmente II und III so breit wie das Distalende der Vorderschienen. Vorderschienen im basalen Drittel des Innenrandes stark erweitert, zum Apex flach bogenförmig ausgeschnitten und stark verengt; die Ausbuchtung dicht bürstenartig behaart. Mittelschienen am Innenrand auf zwei Dritteln der Länge sehr flach ausgeschnitten und dicht mit körnchenartigen Setae besetzt. Aedoeagus mäßig groß; 0,160,19 der Körperlänge messend. Medianlobus im Subapikalbereich ausgeschweift (Fig. 6). Parameren schmal, konvergierend, den Medianlobus etwas überragend.

o: unbekannt.

\section{Differentialdiagnose}

Deliphrosoma slaviankaense sp. n. unterscheidet sich deutlich von Deliphrosoma pirinense ZERCHE, 1991, das im nördlich benachbarten Pirin-Gebirge endemisch ist. Seine Schultern sind viel schmaler [bei pirinense breiter] als das Pronotum. Es hat kleinere Augen und deutlich weiter voneinander entfernte Ocellen als pirinense. Es ist wesentlich heller gefärbt als pirinense, das überwiegend schwarz gefärbt ist; bei pirinense sind nur Schienen und Tarsen schwach aufgehellt. Das Männchen von slaviankaense hat viel stärker verdickte Vorderschienen und viel stärker erweiterte Vordertarsen als pirinense. Der Aedoeagus von slaviankaense ist durch die Ausschweifungen im Subapikalbereich des Medianlobus ausgezeichnet, die bei pirinense und bei allen anderen Arten fehlen, ausgenommen Deliphrosoma tendense ZERCHE, 2001 aus den Meeralpen, wo eine Ausschweifung angedeutet ist. Deliphrosoma angulatum ASSING \& WUNDERLE, 2001 aus dem mittleren und westlichen Griechenland hat viel breitere Schultern als slaviankaense und im Gegensatz zu slaviankaense eine flache Halsfurche, die am besten zwischen den Ocellen erkennbar ist. Die 
Erweiterung der Vorderschienen und -tarsen der Männchen ist bei beiden Arten sehr ähnlich ausgebildet und nicht zur Trennung geeignet.

Deliphrosoma fratellum (ROTTENBERG, 1874) ist meist viel dunkler gefärbt, bei ihm sind die Beine niemals vollständig rotbraun und die Basis der Antennen ist nicht aufgehellt. Das Männchen von fratellum hat viel schwächer erweiterte Vorderschienen.

Die Unterarten von Deliphrosoma prolongatum (ROTTENBERG, 1873) sind schon durch ihre \pm scharfen Hinterwinkel des Pronotums leicht zu unterscheiden.

\section{Verbreitung}

Die neue Art ist nur vom locus typicus in der subalpinen Region des Slavianka-Massivs (= Ali-Botusch) in Südwest-Bulgarien bekannt. Der Fundort ist nur etwa $1 \mathrm{~km}$ von der griechischen Grenze entfernt (griechischer Name: Órvilos).

Derivatio nominis: Den Namen slaviankaense (Adjektiv) wähle ich, weil die neue Art wahrscheinlich im Slavianka-Gebirge endemisch ist.

Untersuchtes Material: $20^{\pi} 0^{*}$.

7. Deliphrosoma prolongatum komense subsp. $\mathrm{n}$. Fig. 3,7

\section{Typenmaterial}

Holotypus ( $\left.\sigma^{\star}\right)$ : BG: Stara Planina, Kom-Massiv, Mali Kom, N-Hang, $1730 \mathrm{~m}$, Schnee-

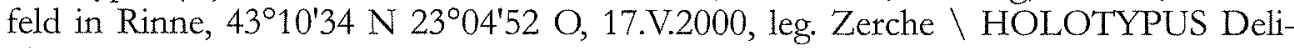
phrosoma prolongatum komense Zerche (DEI).

Paratypen: (1 $\sigma^{\star}, 3$ ㅇ 9$)$ : wie Holotypus, aber: 18.V.2000, leg. Behne (DED).

\section{Beschreibung}

Maße des Holotypus [in mm]: Kopfbreite 0,84; Augenlänge (im Profil) 0,21; Schläfenlänge (im Profil) 0,14; Pronotumlänge 0,76; Pronotumbreite 1,20; Nahtlänge 1,88; Elytrenbreite 1,60; Abdomenbreite 1,46.

Körper weitgehend geschwärzt, Seitenränder und Hinterrand des Pronotums und der Elytren nur sehr schwach aufgehellt, die Elytren nicht heller. Ocellen kaum aufgehellt. Mundteile geschwärzt, nur die Mandibeln etwas heller. Antennen pechbraun, ihre Basis nicht aufgehellt. Beine düster rotbraun, kaum heller als der Körper.

Punktur der Oberseite ziemlich grob, auf dem Kopf etwas feiner als auf dem Pronotum, auf dem Pronotum etwas unregelmäßig, deutlich schwächer als auf den Elytren, hier unregelmäßig gereiht. Mikroskulptur seht schwach ausgebildet, bei 40x auf der Scheibe des Pronotums kaum wahrnehmbar.

Größe: 4,90-5,25 mm ( $\left.\sigma^{7} \sigma^{7}\right), 4,69-5,04 \mathrm{~mm}$ (ㅇ ㅇ) (Mittelwert 4,9 [n = 5]).

Augen groß und vorgewölbt, viel länger als die Schläfen $(1,5)$. Ocellen im Niveau des Augenhinterrandes, voneinander meist nur wenig weiter entfernt als jeweils vom Auge (1,23-1,50; Mittelwert 1,36). Schläfen in flachem Bogen abgerundet, hinter den Augen schwach winkelförmig vorstehend.

Pronotum stark quer $(1,58)$, etwas vor der Mitte am breitesten; nach vorn stärker, nach hinten schwächer, nur schwach ausgeschweift verengt. Vorderwinkel relativ kurz abgerundet. Vorderrand relativ stark doppelbuchtig. Hinterwinkel spitz, fast rechtwinklig. Hinterrand sehr flach konvex. Seitenrandeindrücke tief. Mitte des Pronotums mit einem 


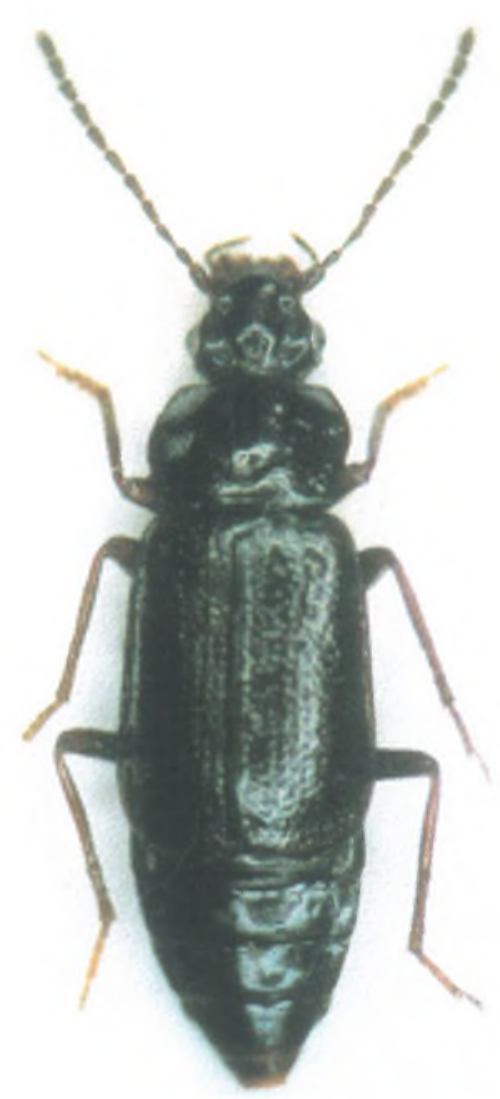

Fig. 3. Deliphrosoma prolongatum komense [Holotypus].

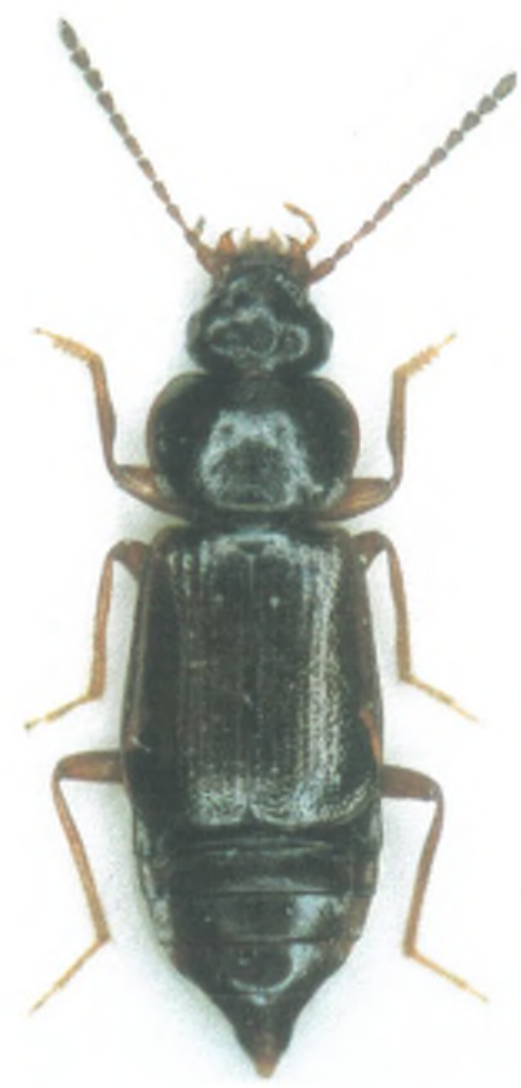

Fig. 4. Deliphrosoma bulgaricum ZERCHE [Midschut-Massiv].

\pm deutlichen strichförmigen Längseindrück und dahinter mit einem stumpfwinkligen bis bogenförmigen Quereindruck (ankerartige Vertiefung).

Elytren gestreckt, mäßig erweitert; ihre Naht deutlich länger als die größte Breite $(1,17)$. Schultern breiter als das Pronotum. Hautflügel entwickelt.

Abdominaltergite V und VI mit je einem Paar kleiner querovaler Tomentflecke, die des Tergits V meist durch die Elytren rerdeckt. Hinterrand des Tergits VII mit sehr schmalem Fransensaum oder ohne einen solchen.

§: Vordertarsen stark erweitert; Segment II so breit wie das Distalende der Vorderschienen. Vorderschienen in der Mitte des Innenrandes etwas winkelförmig erweitert, zum Apex bogenförmig ausgeschnitten und verengt. Mittelschienen kräftiger ausgebildet, am Innenrand auf zwei Dritteln der Länge flach ausgeschnitten und dicht mit kleinen körnchenartigen Setae besetzt. Aedoeagus wie in Fig. 7.

\section{Differentialdiagnose}

Deliphrosoma prolongatum komense subsp. n. ist größer und dunkler gefärbt als die anderen Subspezies. Im Gegensatz zu den anderen Subspezies ist komense weitgehend geschwärzt. 
Seine Elytren sind ziemlich lang, die Naht ist deutlich länger als die größte Breite der Elytren. Seine Ocellen sind durchschnittlich etwas weniger weit getrennt (1,23-1,50; Mittelwert 1,36) als bei den anderen Subspezies $(1,35-1,68)$.
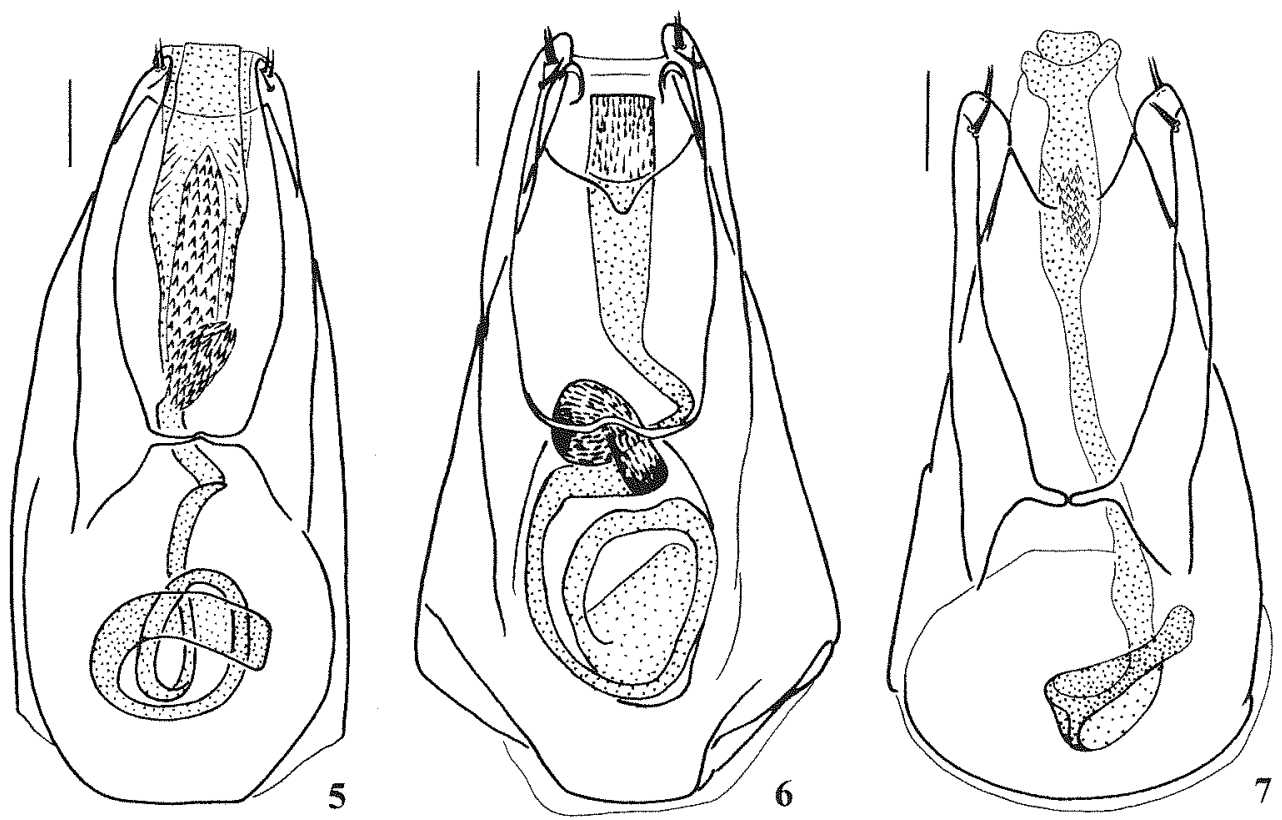

Fig. 5-7. Aedoeagus, ventral [Maßstab 0,1 mm]: Fig. 5. Anthobitm belasizaense sp. n. [Holotypus]. - Fig. 6. Deliphrosoma slaviankaense sp. n. [Holotypus]. - Fig. 7. Deliphrosoma prolongatum komense [Holotypus].

\section{Verbreitung}

Die Subspezies ist bisher nur aus dem Kom-Massiv der Stara Planina bekannt. Die Typen wurden am Rande eines Lawinen-Schneefeldrests in einer steilen Nordrinne gesammelt.

Derivatio nominis: Die Subspezies wird komense (Adjektiv) nach dem Kom-Massiv genannt.

Untersuchtes Material: $2 o^{\pi}, 3$ 우.

\section{Deliphrosoma prolongatum australe ZERCHE, 1998}

Locus typicus: Bulgarien, Nord-Pirin, Hütte Jaworow

\section{Neues Material}

Pirin S Bansko, SO Hütte Banderiza, Straßenrand, $1845 \mathrm{~m}$, unter Pinus mugo am Schnee, $41^{\circ} 46^{\prime} 05$ N $23^{\circ} 25^{\prime} 33$ O, 13.V.2000, 11 o $^{\prime}, 7$ ㅇ \&, leg. Zerche \& Behne (DEI). 


\section{Verbreitung}

Die Subspezies bewohnt die subalpine Region des Pirin-Gebirges. Sie ist sowohl im zentralen Teil des Gebirges, der aus Urgestein aufgebaut ist, als auch im nördlichen Teil, der aus Marmor besteht, vertreten.

Untersuchtes Material: $150^{\star} \sigma^{\top}, 10$ 우 $q$.

\section{Deliphrosoma mandli (SCHEERPELTZ, 1937)}

Locus typicus: Bulgarien, Stara Planina, Massiv des Masalat

\section{Neues Material}

Stara Planina, Kom-Massiv, Mali Kom, N-Hang, $1730 \mathrm{~m}$, Schneefeld in Rinne, 43⒈'34 N $23^{\circ} 04^{\prime} 52$ O, 17.V.2000, leg. Zerche, 1 o $^{*}$ (DEI); Stara Planina, Weschen, N-Seite, $1810 \mathrm{~m}$, Schnee in Lawinentinne, $42^{\circ} 45^{\prime} 50$ N 24 $23^{\prime} 54$ O, 24.V.2000, leg. Zerche \& Behne, $5 o^{\star} 0^{\prime \prime}$, 4 우우 (DEI).

\section{Verbreitung}

Deliphrosoma mandli war aus Bulgarien nur aus dem Botev-Masalat-Massiv in der zentralen Stara Planina und vom Vitoscha-Gebirge bekannt. Die Art ist aber in der Stara Planina weiter verbreitet; sie wird hier zum ersten Mal aus dem westlich vom Botev-Massiv liegenden Weschen-Massiv und aus dem viel weiter westlichen Kom-Massiv gemeldet.

Untersuchtes Material: 21 ô $^{\star}, 12$ 우.

\section{Deliphrosoma bulgaricum ZERCHE, 1998}

Fig. 4

Locus typicus: Bulgarien, Rila-Gebirge, SW Ribni Ezera

\section{Neues Material}

Stara Planina, Midschur-Massiv, N-Seite, S Gorni Lom, Schneerinne, $1640 \mathrm{~m}$, Gras, Vaccinium, $43^{\circ} 24^{\prime} 05$ N 22 $42^{\prime} 53$ O, 21.V.2000, leg. Zerche, 8 o ơ, 13 ㅇ + (DEI); Stara Planina, Midschur-Massiv, N-Seite, S Gorni Lom, Schneerinne, $1675 \mathrm{~m}$, Juniperus, Vaccinium, $43^{\circ} 24^{\prime} 05$ N 22 $42^{\prime} 53$ O, 21.V.2000, leg. Zerche, 6 ơ o 8 우 우 (DEI); Stara Planina, Midschur-Massiv, N-Seite, S Gorni Lom, Schneerinne, $1750 \mathrm{~m}, 43^{\circ} 24^{\prime} 03 \mathrm{~N} 22^{\circ} 42^{\prime} 51 \mathrm{O}$, 21.V.2000, leg. Behne, 1 o (DEI).

\section{Variabilität}

Die Differentialmerkmale der Originalbeschreibung, die nur auf dem einzelnen Holotypus basierten, haben sich im vollen Umfang bestätigt. Ektoskelettal stimmen alle 36 neu aufgefundenen Tiere mit dem Holotypus überein, die untersuchten Männchen entsprechend im Genitalbau. Die Größe schwankt zwischen 4,20 und 5,74 mm.

\section{Verbreitung}

Bisher war nur der locus typicus im Rila-Gebirge bekannt. Die Art wird hier zum ersten Mal aus dem Midschur-Massiv gemeldet, dem nordwestlichsten alpinen Massiv der Stara Planina. Die Art dürfte auch im serbischen Teil dieses Bergmassivs vorkommen. Alle neu gesammelten 36 Tiere stammen aus einer einzigen Schneerinne.

Untersuchtes Material: $160^{\star} 0^{\star}, 21$ 우. 


\section{Anmerkungen zu Deliphrosoma angulatum ASSING \& WUNDERLE, 2001}

\section{Verbreitung und Material}

Die Art ist im südlichen Mittelgriechenland und in Westgriechenland weiter verbreitet als in der Beschreibung angegeben. Sie wird hier auch aus dem Iti-, dem Peristeri- und dem Timfi-Massiv gemeldet. Mir liegt das folgende Material vor:

GR: Fokida, Óri Vardoússia, W Athanasios-Diakos, Schnee im Rasen, N-Hang, $1710 \mathrm{~m}$, $38^{\circ} 41^{\prime} 52$ N 22 $08^{\prime} 44$ O, 5.V.1999, leg. Zerche, 4 o o $0^{\star}, 2$ 우 (DEI); GR: Fokida, Óri Vardoússia, W Athanasios-Diakos, unter Steinen, N-Hang, $1710 \mathrm{~m}, 38^{\circ} 41^{\prime} 52 \mathrm{~N} 22^{\circ} 08^{\prime} 44 \mathrm{O}$, 5.V.1999, leg. Behne, 1 \& (DEI); GR: Fthiótida, Parnassos (Ski-Center), Schnee auf Schotter, N-Hang, $2030 \mathrm{~m}, 38^{\circ} 23^{\prime} 58 \mathrm{~N} 22^{\circ} 34^{\prime} 57$ O, 7.V.1999, leg. Zerche, 1 o*, 1 o (DEI); Zentral-GR, Parnassosgebirge, 2250, Gesiebe, Gräser, Schneefeeldrand, 11.6.1996, P. Wunderle, $20^{*} o^{*}$ (DEI); GR: Fthiótida, Óros Iti, SO Kastania, N-Hang S Bergsattel, unter Steinen am Schnee, $2010 \mathrm{~m}, 38^{\circ} 48^{\prime} 07$ N 2215'14 O, 6.V.1999, leg. Zerche, $10^{\circ}, 1$ 우 (DEI); GR Epirus [3724], Peristeri Mts. b. Anthohori, 1700-2200 m, 39.41/21.07, leg. Marggi, 4.6.2000, $220^{\star} o^{\star}, 15$ ㅇ + (DEI; coll. Marggi); GR Konitsa, Timfi Mts., Schutzhütte - Gipfel, 39.58+20.47, 29.5.2000, leg. W. Marggi, 1 우 (DED).

\section{Differentialdiagnose}

Die Art ist Deliphrosoma fratellum sehr ähnlich. Sie ist aber heller gefärbt. Frisches Material ist in der Regel schon an der Färbung zu unterscheiden; altes Museumsmaterial von fratellum einschließlich der Typen ist aber ähnlich hell wie angulatum. Neben den stärker und etwas winkelförmig verdickten Vorderschienen des Männchens ist das sicherste Unterscheidungsmerkmal eine flache Halsfurche, die zwischen den Ocellen deutlich zu erkennen ist und auch die Trennung der Weibchen ermöglicht. Dieses Merkmal ist bei allen 51 untersuchten Exemplaren vorhanden und fehlt bei allen 63 untersuchten fratellum-Exemplaren einschließlich der Lectotypen von Lathrimaeum fratellum ROTTENBERG, 1874 und des Synonyms Arpediwm major BERNHAUER, 1902. Die Abbildung des Aedoeagus in der Beschreibung ist nicht ganz vollständig; auch angulatum hat eine parabelförmige bis fast V-förmige Einbuchtung im Apikalteil der Medianlobus sowie den Ansatz der Parameren viel weiter herunter gezogen und entspricht in diesen Merkmalen jeweils fratellum. Der Aedoeagus weist nur geringfügige Unterschiede auf und ist zur Trennung kaum geeignet.

Für die spezifische Verschiedenheit von fratellum und angulatum spricht auch die Tatsache, dass sie in vier Gebirgen (Vardousia, Parnassos, Peristeri, Timfi) gemeinsam vorkommen. Ihre Areale überschneiden sich also weiträumig.

Untersuchte Exemplare: 30 ठ゚

\section{Anmerkungen zu Deliphrosoma fratellum (ROTTENBERG, 1874)}

\section{Verbreitung und Material}

Deliphrosoma fratellum ist auch in Mittel- und Westgriechenland verbreitet, im Nordosten reicht ihr Areal bis zum Olymp, im Nordwesten bis nach Serbien und Montenegro. Zur Trennung von angulatum siehe dort (Differentialdiagnose). Mir liegt das folgende, noch nicht publizierte, Material vor:

GR: Peloponnes, Ahaïa, Panahaiko-Geb., N-Seite, $1610 \mathrm{~m}, 38.11 .00 \mathrm{~N} 21.51 .41 \mathrm{O}$, 29.IV.1998, Schneefelder, leg. Zerche, 1 \& (DEI); GR: Peloponnes, Ahaïa, Chelmos- 
Geb. O Kalávrita, O Xerokambos, N-Hang, 1710 m, 38.00.20 N 22.11.55 O, 27.IV.1998, lichter Tannenwald, Schneefelder, leg. Zerche, 2 o + (DEI); GR: Peloponnes, Korinthía, Killini-Geb. S Trikala, 2080 m, 37.55.41 N 22.24.09 O, 28.IV.1998, zw. Fels und Schneefeld, leg. Zerche, 10 o o $^{*} 7$ ㅇ 9 (DEI); GR: Peloponnes, Korinthía, Killini-Geb. S Trikala, 1890 m, 37.55.48 N 22.24.45 O, 24.IV.1998, Schneefelder in Bachschlucht, leg. Zerche, $2 o^{*}$ (DEI); GR: Peloponnes, Korinthía, Killini-Geb. S Trikala, $1770 \mathrm{~m}, 37.55 .45$ N 22.25.20 O, 24.IV.1998, unter Steinen, leg. Behne, 1 ㅇ (DEI); GR: Peloponnes, Korinthia, Killini-Geb., S Ano Trikala, Schnee im Rasen, O-Hang, $2010 \mathrm{~m}, 37^{\circ} 55^{\prime} 53 \mathrm{~N}$ $22^{\circ} 24^{\prime} 06$ O, 1.V.1999, leg. Zerche \& Behne, $10^{*}, 2$ ㅇ $q$ (DEI); GR: Fthiótida, Parnassos, oberh. Ski-Center, 1965 m, 38.32.30 N 22.35.31 O, 25.IV.1998, Schneerand, unter Juniperus, leg. Zerche, 2 q $q$ (DEI); GR: Fokida, Óri Vardoússia, W Athanasios-Diakos, Schnee im Rasen, N-Hang, $1710 \mathrm{~m}, 38^{\circ} 41^{\prime} 52$ N 22008'44 O, 5.V.1999, leg. Zerche, $10^{\circ}$ (DEI); GR Epirus [3724], Peristeri Mts. b. Anthohori, 1700-2200 m, 39.41/21.07, leg. Marggi, 4.6.2000, 10, $1 \%$ (DEI); GR Konitsa, Timfi Mts., Schutzhütte - Gipfel, 39.58+20.47, 29.5.2000, leg. W. Marggi, 3 o o , 1 \% (DEI; coll. Marggi); GR, Epirus, Smolikas Mts., 2200-2500 m, 40.05+20.57 [3727], 31.5.2000, leg. Marggi, 2 ơ o , 4 q o (DEI; coll. Marggi); GR, Lithochoron, Mt. Olympus, 2500 m, 40.05+22.21, 4.6.1999, leg. Marggi/Huber, 2 우 (DEI).

\section{Katalog der Deliphrosoma-Arten und -Unterarten Bulgariens}

bulgaricum ZERCHE, 1998

mandli (SCHEERPELTZ, 1937)

slaviankaense sp. $\mathbf{n}$.

pirinense ZERCHE, 1991

prolongatum australe ZERCHE, 1998 Pirin-Gebirge

prolongatum rbilense ZERCHE, 1998 Rila-Gebirge

prolongatum balcanicum ZERCHE, 1998 Balkan-Gebirge (Botev-Massiv)

prolongatum komense subsp. n. Balkan-Gebirge (Kom-Massiv)

prolongatum vitoschaense ZERCHE, 1998 Vitoscha-Gebirge
Rila-Gebirge, Balkan-Gebirge (Midschur-Massiv)

Balkan-Gebirge (Kom-Massiv, Weschen-Massiv, Botev-und Masalat-Massiv), Vitoscha-Gebirge; [Türkei: Nordwest-Anatolien (Uludağ, Ilgazdağ)] Slavianka-Gebirge $[=$ Ali-Botusch $=$ Órvilos $]$ Pirin-Gebirge

\section{Literatur}

Assing, V. \& WUNDERLE, P. 2001: On the Staphylinidae of Greece. II. New species and new records from central and northern Greece (Insecta: Coleoptera). - Linzer biol. Beitr. 33 (1): 103-136.

HAMmOND, P. M. 1972: The micro-structure, distribution and possible function of peg-like setae in male Coleoptera. - Ent. scand., Lund 3 (1): 40-54.

HoRION, A. 1963: Faunistik der mitteleuropäischen Käfer. Bd. IX: Staphylinidae. 1. Teil. Micropeplinae bis Euaesthetinae. - Überlingen-Bodensee: XII + $412 \mathrm{~S}$.

LUZE, G. 1905: Revision der paläarktischen Arten der Staphyliniden-Gattung Latbrimaeum ER. Verh. zool.-bot. Ges. Wien 55: 53-69 
SCHEERPEITZ, O. 1937: Wissenschaftiche Ergebnisse einer von Herrn Hofrat F. SCHUBERT, seinem Sohne Herrn cand. phil. F. SCHUBERT und Herrn Prof. Ing. K. MANDL im Sommer 1935 (1936) nach Bulgarien unternommenen Studienreise. Coleoptera: I. Staphylinidae. (24. Beitrag zur Kenntnis der paläarktischen Staphyliniden, gleichzeitig 1. Beitrag zur Kenntnis der Staphyliniden Bulgariens). - Mitt. Königl. Naturwiss. Inst., Sofia 10: 185-246.

SCHEERPELTZ, O. 1961: Neue Arten der Gattung Latbrimaeum ER., nebst einer Bestimmungstabelle der bisher bekannt gewordenen palaearktischen Arten dieser Gattung (Col. Staphylinidae). (108. Beitrag zur Kenntnis der paläarktischen Staphyliniden). - Mitt. Münchner Ent. Ges. 51: 72-95.

SCHEERPELTZ, O. 1963: Ergebnisse der von WILHELM KüHNELT nach Griechenland unternommenen zoologischen Studienreisen. I. (Coleoptera-Staphylinidae). (124. Beitrag zur Kenntnis der paläarktischen Staphyliniden). - Sitzber. Österr. Ak. Wiss., Mathem.-naturw. Kl., Abt. I, 172 (9-10): 413-452.

ZERCHE, L. 1991 a: Beitrag zur Taxonomie und Verbreitung der Gattung Deliphrosoma REITTER, 1909 (Coleoptera, Staphylinidae, Omaliinae). - Beitr. Ent., Berlin 41 (2): 313-332.

ZeRCHE, L. $1991 \mathrm{~b}$ : Zur Taxonomie und Verbreitung der Gattung Delipbrosoma RerrTer (Staphylinidae: Omaliinae). - Verh. Westd. Entom. Tag 1990, Düsseldorf: 147-153.

ZERCHE, L. 1997: Eine neue Art der Gattung Deliphrosoma aus der Nordost-Türkei und Ergänzungen zur Verbreitung der Gattung (Insecta: Coleoptera: Staphylinidae: Omaliinae). - Reichenbachia Mus. Tierkd. Dresden 32 (9): 49-52.

ZERCHE, L. 1998: Neue Deliphrosoma-Arten aus Bulgarien und aus der Türkei und neue Unterarten von Deliphrosoma prolongatum (ROTTENBERG) aus Bulgarien (Insecta: Coleoptera: Staphylinidae: Omaliinae). Reichenbachia Mus. Tierkd. Dresden 32 (36): 245-255.

ZERCHE, L. 1999: Biodiversitätsforschung in Europa am Beispiel bulgarischer Hochgebirge (Coleoptera: Staphylinidae, Leiodidae). - Cour. Forsch.-Inst. Senckenberg, Frankfurt a. M. 215: 233-238.

ZeRCHE, L. 2001: Eine neue Art der Gattung Delipbrosoma aus den Meeralpen (Insecta: Coleoptera: Staphylinidae: Omaliinae). - Reichenbachia Mus. Tierkd. Dresden 34 (1): 119-122.

\section{Anschrift des Verfassers:}

Dr. LOTHAR ZERCHE

Deutsches Entomologisches Institut

im Zentrum für Agrarlandschafts- und

Landnutzungsforschung (ZALF)

Schicklerstraße 5

D-16225 Eberswalde

Deutschland

e-mail: zerche@zalf.de 